\title{
Die Art der Krebstherapie beeinflusst die Impfantwort
}

Krebspatienten haben ein erhöhtes Risiko für SARS-CoV-2-Infektionen und schwere COVID-19-Verläufe. Die Corona-Impfung ist generell für Krebspatienten empfohlen, allerdings weiß man bisher wenig darüber, wie gut diese auf die Impfung ansprechen. In einer Studie in JAMA Oncology konnte ein interdisziplinäres Team der MedUni Wien unter Leitung von Prof. Dr. Matthias Preusser zeigen, dass die Art der Krebstherapie die Impfantwort beeinflusst: Patienten unter Chemotherapie hatten geringere Antikörperspiegel als Patienten unter zielgerichteter medikamentöser Therapie bzw. bei Injektion oder Infusion.

Quelle: Presseaussendung der Medizinischen Universität Wien
Die Wissenschaftler der Universitätsklinik für Innere Medizin I sowie vom Klinischen Institut für Labormedizin von MedUni Wien und AKH Wien und der hämato-onkologischen Abteilung des Krankenhauses Meran untersuchten bei über 600 Teilnehmern in zwei Patientengruppen und in einer Kontrollgruppe aus gesundem Spitalspersonal die Antikörperbildung nach der Corona-Impfung [1]. Preusser: „Dabei wurden sowohl Patienten und Patientinnen mit soliden Krebserkrankungen als auch Patienten mit Blutkrebserkrankungen eingeschlossen."

Das zentrale Ergebnis: Die Form der Krebstherapie beeinflusst die Impfantwort. Dr. Maximilian Mair von der Klinischen Abteilung für Onkologie, Erstautor der Studie, erläutert: „Patientinnen und Patienten unter Chemotherapie hatten geringere Antikörperspiegel als Patienten unter zielgerichteterTherapie. Die geringsten Antikörperspiegel fanden wir bei Patienten mit Blutkrebserkrankungen, die eine zielgerichtete Therapie gegen bösartige B-Zellen erhalten hatten."

\section{Niedrigere Antikörperspiegel}

Sowohl bei gesunden Personen als auch bei Krebspatienten waren die Antikörperspiegel nach Vollimmunisierung mit den zugelassenen CoronaImpfstoffen höher als nach nur einer Impfdosis, aber die Antikörperspiegel waren selbst nach Vollimmunisierung bei gesunden Personen höher als bei Krebspatienten. Unterschiede zwischen verschiedenen Impfstoffen konnten nicht nachgewiesen werden, wobei die meisten Personen einen mRNA-Impfstoff erhalten hatten.

Preusser: „Insgesamt zeigen unsere Daten, dass die meisten Krebspatienten nach Impfung Antikörper gegen das SARS-CoV-2 Spike Protein entwickeln und daher die Impfung generell empfohlen ist, dass aber der Impfschutz durch manche Krebstherapien abgeschwächt wird. Allgemeine Schutzmaßnahmen wie regelmäßige CoV-
Testungen und Hygienemaßnahmen erscheinen daher gerade bei Krebspatienten unter laufender Krebstherapie selbst nach Impfung sehr wichtig, um einen optimalen Schutz vor COVID-19 zu erzielen."

\section{Literatur}

1. Mair MJ et al Humoral immune response in hematooncological patients and health care workers who received SARS-CoV-2 vaccinations. JAMA Oncol. https://doi.org/10.1001/ jamaoncol.2021.5437

Hinweis des Verlags. Der Verlag bleibt in Hinblick auf geografische Zuordnungen und Gebietsbezeichnungen in veröffentlichten Karten und Institutsadressen neutral.

hautnah $2021 \cdot 20: 192-193$ https://doi.org/10.1007/s12326-02100472-4

C Springer-Verlag GmbH Austria, ein Teil von Springer Nature 2021 
Hier steht eine Anzeige.

Springer 